\title{
Le dialogue complexe entre le national et l'international dans la poésie d'avant-garde finlandaise des années 1960
}

The complex dialogue between the national and the international in Finnish avant-garde poetry of the 1960s

Kansallisen ja kansainvälisen monitahoinen dialogi 1960-luvun suomalaisessa avantgarderunoudessa

\section{Harri Veivo}

\section{OpenEdition}

Journals

Édition électronique

URL : https://journals.openedition.org/efo/148

DOI : $10.4000 /$ efo. 148

ISSN : 2275-1947

Éditeur

INALCO

Édition imprimée

Date de publication : 1 janvier 2011

ISBN : 978-2-296-96641-3

ISSN : 0071-2051

Référence électronique

Harri Veivo, «Le dialogue complexe entre le national et l'international dans la poésie d'avant-garde finlandaise des années 1960 », Études finno-ougriennes [En ligne], 43 | 2011, mis en ligne le 05 mars 2014, consulté le 08 juillet 2021. URL : http://journals.openedition.org/efo/148 ; DOI : https://doi.org/ 10.4000/efo. 148

Ce document a été généré automatiquement le 8 juillet 2021.

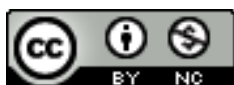

Études finno-ougriennes est mis à disposition selon les termes de la Licence Creative Commons Attribution - Pas d'Utilisation Commerciale 4.0 International. 


\title{
Le dialogue complexe entre le national et l'international dans la poésie d'avant-garde finlandaise des années 1960
}

\author{
The complex dialogue between the national and the international in Finnish \\ avant-garde poetry of the 1960s \\ Kansallisen ja kansainvälisen monitahoinen dialogi 1960-luvun suomalaisessa \\ avantgarderunoudessa
}

Harri Veivo

Selon la légende, le violoniste Arvo Hannikainen aurait été le premier poète dadaïste de Finlande. Vers le début des années vingt, à Paris, dans un bar après minuit, il aurait récité, d'une voix dramatique et retentissante, un poème constitué par la série des nombres de yksi à kymmenen (Paavolainen 2002, p. 93-94). Malgré le grand succès que ce poème-événement aurait connu, le dadaïsme et les autres mouvements d'avant-garde sont restés marginaux dans la littérature finlandaise jusqu'à la fin des années cinquante. Le recueil d'essais Nykyaikaa etsimässä (À la recherche du contemporain, 1929) d'Olavi Paavolainen et quelques poèmes d'Aaro Hellaakoski et des écrivains suédophones Gunnar Björling et Rabbe Enckell mis à part, la littérature finlandaise a préféré chercher l'inspiration d'abord dans le vitalisme et l'expressionnisme germanique des années vingt, puis dans le modernisme anglo-américain de T.S. Eliot et Ezra Pound dans les années cinquante ${ }^{1}$. En revanche, les années soixante voient naître un nouvel intérêt pour le dadaïsme et le surréalisme, ainsi que pour les mouvements contemporains d'expérimentations littéraires comme la poésie concrète, le lettrisme, la Beat generation et le Pop Art. La "nouvelle poésie » finlandaise s'empare de l'héritage de Guillaume Apollinaire, de Tristan Tzara et d'André Breton et suit de près le travail d'écrivains contemporains comme Allen Ginsberg, Carl Fredrik Reutersvärd et Helmut Heißenbüttel. D'un coup, de nouveaux noms d'écrivains, de mouvements artistiques et de titres d'œuvres apparaissent dans les journaux et les revues littéraires ainsi que 
dans les textes qui cherchent à renouveler le langage poétique en utilisant des techniques avant-gardistes comme le collage, le montage, le ready-made et la mise en page dynamique et « libre».

Que signifie cette apparition brusque de nouveaux intertextes et de nouvelles pratiques poétiques, cette réorientation des points cardinaux de la poésie finlandaise sur l'atlas de la littérature mondiale ? Dans cet article, je vais essayer de montrer que ce dialogue avec l'avant-garde historique et la littérature expérimentale contemporaine est un phénomène à facettes multiples, dont la complexité va bien au-delà de la simple arrivée tardive de nouvelles idées dans un pays lointain qui souffre du décalage de la périphérie par rapport au centre. Il s'agit plutôt d'un processus dans lequel la poésie finlandaise cherche à sauvegarder une partie de la force critique de l'avant-garde historique et contemporaine, tout en l'adaptant au contexte local et en négociant sa position au sein d'une société de consommation et du spectacle émergente ${ }^{2}$. La poésie finlandaise des années soixante peut être qualifiée de "néo-avant-garde», mais uniquement dans le sens que Harold Foster a donné à cette notion, comme un travail d'échange, d'anticipation et de reconstitution dans un espace-temps diagonal de l'avant-garde (Foster 1994). L'intérêt que les poètes de l'époque ont porté aux avantgardes historiques témoigne de l'internationalisation de la culture et de la société finlandaises, mais il est également motivé par un jeu de positionnement à l'intérieur du champ littéraire national qui connaît au cours de la décennie une série de mutations fondamentales conduisant à la professionnalisation du statut de l'écrivain et à la redéfinition des genres et des limites du discours littéraire, notamment par rapport à la religion et aux mœurs. Ces mutations sont inséparables d'une conscience temporelle spécifique et d'une véritable «culture de crise » (Calinescu, 1987, p. 124) partagée par de nombreux écrivains et autres acteurs du champ littéraire. Le présent n'était plus vu en relation de continuité avec le passé, mais en rupture avec lui, soumis à un questionnement permanent motivé par la transformation intégrale et rapide de la société où la permanence du changement était la seule certitude. Dans ce contexte, l'expérimentation artistique, même au risque d'un échec, était souvent plus importante que la recherche de résultats durables.

Pour retracer cette évolution, je vais d'abord me concentrer sur la transformation progressive du discours poétique au cours des années cinquante et soixante et sur le sentiment de crise qui caractérise «la nouvelle poésie» et qui motive l'importance accordée au dialogue international. Ensuite, j'aborderai la question de l'appropriation et de l'interprétation des avant-gardes historiques et étrangères dans le travail du poète, essayiste et traducteur Väinö Kirstinä, qui fut l'une des figures marquantes de la décennie. La carrière de Kirstinä permet de discerner au niveau de l'individu des structures et des motivations d'ordre plus général qui entrent en jeu dans le dialogue transnational entre l'avant-garde internationale et la littérature finlandaise.

\section{La culture de crise et l'élargissement des horizons dans les années soixante}

Dans la poésie finlandaise, la génération des années cinquante, souvent appelée «la deuxième vague du modernisme » (après celle de la fin des années vingt), a cherché à épurer le discours poétique des archaïsmes, des structures métriques jugées artificielles et des figures de style ornementales et pesantes. Dans cette recherche, elle s'est 
inspirée du modernisme et du new criticism anglo-américain, dont les figures de proue comme T.S. Eliot (prix Nobel 1948) et Ezra Pound sont vite devenues des autorités incontestables aux yeux des jeunes poètes et prosateurs finlandais (Kantola et Riikonen, 2007, p. 452-457). Pour les modernistes des années cinquante, la poésie devait être exacte, claire et concrète. Cette aspiration était en même temps esthétique et éthique. Le discours poétique devait être lucide et prêt à confronter des points de vue et des regards différents, voire opposés. En même temps, les poètes cherchaient à aménager pour la littérature et l'art une position autonome par rapport aux discussions sociales et poétiques. Selon le poète et éditeur Tuomas Anhava, autorité esthétique de cette génération, l'évolution de la langue se faisait essentiellement dans la littérature, qui avait en conséquence une place centrale dans la culture d'une nation. Cette place exigeait une dévotion absolue: un homme de lettres devait "manger et boire de l'encre, penser à l'encre et rêver de l'encre » (cité dans Suurpää, 2008, p. 61-62; voir aussi Hökkä, 1999, p. 76-81).

5 La poésie des années cinquante contient déjà les germes d'une grande partie des contradictions et des aspirations opposées qui vont évoluer vers une véritable crise de la culture au cours de la décennie suivante. Elle croyait au progrès dans la littérature, la culture et la société, et considérait que l'art avait un rôle fondamental à jouer dans cette évolution. Cette foi typiquement moderne était accompagnée d'un intérêt pour la grande tradition de la littérature mondiale, qui s'étendait des écrivains modernes comme Virginia Woolf aux auteurs classiques comme Andrew Marvell et Cervantès et au-delà à la poésie folklorique finlandaise, la littérature classique du Japon et les écrivains de l'antiquité grecque et romaine (Viikari, 1992, p. 37-38; Suurpää, 2008, p. 62-63). Le renouvellement du langage poétique contemporain était accompagné d'un projet conservateur, respectueux du canon. D'autre part, la poésie des années cinquante aspirait à la raison et au libéralisme de l'esprit et cherchait un point de vue lucide et éthique par rapport à la société, mais elle n'a pas voulu entrer dans une discussion directe avec les grandes questions sociales ou les idéologies politiques de l'époque. À l'engagement et au combat, elle a préféré l'observation et la figure du « no man's land» (Viikari, 1992).

6 L'héritage le plus important de la poésie des années cinquante reste cependant le fait qu'elle a installé une série d'attentes à l'égard de la poésie et de la littérature, ainsi qu'un modèle évolutif pour penser son histoire et son état présent. À la fin des années cinquante et dans les années soixante, la poésie et la littérature sont considérées comme des phénomènes historiques, c'est-à-dire soumis à des changements, propres à un travail de perfectionnement, mais aussi à des processus de dégénérescence. Cette façon de penser était promue par la deuxième vague du modernisme, qui a entamé le travail d'épuration et d'amélioration du discours poétique et qui a voulu établir une nette rupture par rapport à la poésie de l'avant-guerre. Aux yeux du public, et dans les colonnes des revues et journaux, elle se traduit par une interrogation sur le sens de l'évolution. Le public attend des changements, l'apparition du nouveau, l'essor d'une nouvelle génération. La littérature, et avant tout la poésie, est pensée en fonction de la société actuelle et de son avenir. En témoignent les titres de communications et d'articles tels que «La poésie des années soixante, ce qu'elle sera » et «La poésie des années soixante, ce qu'elle devra être " d'Osmo Hormia et d'Arvo Salo, prononcées d'abord au séminaire de poésie de Turku les 29 et 30 septembre 1962 et publiés ensuite dans les actes du séminaire et dans la revue Parnasso, ou bien les réponses par Pentti 
Saarikoski et Salo à la question "Qu'attendez-vous de notre littérature des années soixante? ? dans les rubriques de la même revue au premier numéro de l'année 1960 : "Je suis toujours darwinien" (Saarikoski, 1960, p. 8) et "Qu'elle laisse vite dans l'ombre tous les résultats des années cinquante » (Salo, 1960, p. 9) ${ }^{3}$.

7 Le discours littéraire du début des années soixante s'inscrit donc d'une manière particulière dans la continuité avec la décennie précédente. Comme de nombreux essayistes et chercheurs contemporains l'ont plus tard montré, il n'y a pas de rupture véritable, mais plutôt une continuité dans la recherche de renouvellement (Anhava, 1967, Polameri, 1969, Haapala, 2007). Mais en même temps, le modernisme des années cinquante devient vite un succès artistique et même commercial, une sorte de nouveau canon, que les jeunes poètes et critiques commencent à accuser d'immobilisme, de stagnation, de clôture (Kirstinä, 1962a, Peltomäki, 1963). Les jeunes poètes qui ont publié leurs premiers textes à la fin des années cinquante ou au début de la décennie suivante partagent une prise de conscience évidente, mais diffuse de la nécessité d'avancer, de chercher de nouvelles manières d'écrire qui répondent mieux à l'évolution de la société.

8 Une solution pour décloisonner le discours poétique était offerte par l'internationalisation, par l'ouverture dans un premier temps vers la tradition européenne de la poésie moderne et ensuite vers les avant-gardes historiques et la poésie contemporaine de la Beat generation et de l'Amérique latine. ${ }^{4}$ Les années soixante présentaient de plus en plus d'occasions de rencontrer des écrivains étrangers en Finlande, par exemple lors du Festival international de la jeunesse et des étudiants, en 1962, et des conférences bisannuelles de Lahti à partir de 1963. La publication de traductions, d'anthologies et d'essais sur la poésie étrangère témoigne bien de cette recherche d'ouverture. La liste suivante, très loin d'être exhaustive, donne une idée des changements en cours pendant les années soixante (essais et poèmes entre guillemets, recueils et anthologies en italiques):

1957, Jaakko AHоKAS : « Kääntämättömiä ranskalaisia » (« Des français qui n’ont pas été traduits ", mentionne par exemple Jarry, Apollinaire, Reverdy, Tzara, Breton, Eluard, Leiris).

1959, Anselm Hollo: "Lyhyt beaattinen anthologie " («Une brève anthologie beat ", poèmes de Ginsberg, Corso et Ferlinghetti, entre autres).

1960, Mirja BolgÁr : "Uuden romaanin sokkelossa» («Dans le labyrinthe du nouveau roman », mentionne par exemple Robbe-Grillet, Beckett, Sarraute).

1961, Allen GINSBERG : Huuto (Howl), traduit par Anselm Hollo.

1961, John CAGE : Määräämättömyys (Indeterminacy), traduit par Ilpo Sainio.

1962, Tulisen järjen aika (L'âge de la raison ardente, poèmes d'Apollinaire, Breton, Aragon, Queneau, etc.), traduit par Aale Tynni.

1962, Helmut HEISSENBÜTTEL : Textbuch II, traduit par Ilkka-Juhani Takalo-Eskola et Paul Glückert.

1963, Allen GINSBERG : Kuolema van Goghin korvalle (Death to van Gogh's Ear), traduit par Pentti Saarikoski, Anselm Hollo et Matti Rossi.

1963, Väinö KIRSTINÄ : « Loruista lettrismiin » (« Des comptines au lettrisme », sur le dadaïsme, le concrétisme, le lettrisme).

1963, Idän ja lännen runot (Les poèmes de l'est et de l'ouest, poèmes de Voznessenski, Ahmadulina, Corso, Ferlinghetti, Olson, etc.), traduit par Markku Lahtela et Anselm Hollo.

1964, Tristan TZARA : série de poèmes, traduits par Jaakko Ahokas.

1964, Väinö KIRSTINÄ : «Kunnianosoitus Tristan Tzaralle » ( Hommage à Tristan Tzara »). 
1964, Väinö KIRSTINÄ : « Näkyvien runojen koulukunta, konkretismi » (“L'école de la poésie visuelle, le concrétisme").

1964, Alain ROBBE-GRILLET : Labyrintissa (Dans le labyrinthe), traduit par Olli-Matti Ronimus et Pentti Holappa.

1964, Näin ihminen vastaa (C'est ainsi que l'homme répond, poèmes de Paz et Vallejo, entre autres).

1965, Tom SANDELL : «Den konkreta poesin - metoden och möjligheter » (« La poésie concrète - méthodes et possibilités ", mentionne par exemple Öyvind Fahlström, Carl Fredrik Reutersvärd).

1965, Hans ARP : deux poèmes, traduits par Erkki Aura.

1966, Guillaume APOLLINAIRE : «Il pleut », « La colombe poignardée et le jet d'eau », traduits par V. J. Polameri.

1966, J. G. Weightman : «Ranskan kirjallisuuden näköaloja » («Horizons de la littérature française », mentionne par exemple Le Clézio, Sollers, Tel Quel), traduit par Auli Tarkka.

1967, Sepро Тчокко: «Muistokirjoitus surrealismille ja Bretonille» («Discours commémoratif du surréalisme et de Breton »).

1969, Kello 0 ( 0 heures, poèmes de Cardenal, Asturias, Paz, entre autres), traduit par Pentti Saaritsa.

1970, André BRETON : Surrealismin manifesti (Le manifeste du surréalisme), traduit par Väinö Kirstinä.

1970, Guillaume APOLLINAIRE : «Vyöhyke » (« Zone »), traduit par Veikko Polameri.

En premier lieu, la liste met en évidence la volonté de suivre de près les expérimentations et les discussions contemporaines. Le nouveau roman français et le travail du groupe Tel Quel sont présentés au public finlandais au fur et à mesure qu'ils font leur percée en France - Paris restera d'ailleurs le lieu de passage ou de séjour préféré des écrivains finlandais tout au long de la décennie. L'intérêt pour le travail de Helmut Heißenbüttel, de John Cage et des concrétistes suédois s'inscrit dans cette logique de suivi de l'expérimentation formelle contemporaine, tandis que les traductions d'Allen Ginsberg - qui provoquent des scandales - montrent aussi la volonté de questionner la société, d'explorer de nouvelles formes de vie ${ }^{5}$. La communication ne se fait pas seulement dans un sens unique, mais sous forme de réseau à plusieurs partenaires, comme le montrent par exemple la traduction de l'essai en anglais de J. G. Weightman (avec tout son travail d'interprétation et d'évaluation) sur la littérature française et l'anthologie Idän ja lännen runot qui cherchait à construire un pont entre les camps opposés de la guerre froide.

La sortie de l'anthologie Tulisen järjen aika - sous-titrée «Dix poètes modernes français »- est le premier effort à grande échelle pour rendre accessible au public finlandais l'héritage de la poésie moderne et d'avant-garde du début du siècle, mais sa réception suscite une vive discussion sur la réussite de l'opération, plusieurs poètes reprochant à Aale Tynni d'avoir publié des traductions "muséales» et non pas vivantes. L'anthologie est suivie d'une série de traductions de textes individuels qui essaient de corriger cette erreur, avec en point d'orgue la parution du Manifeste $d u$ surréalisme en finnois en 1970. Ensuite, il apparaît clairement que, à l'exception de l'anthologie de Tynni et des contributions de Jaakko Ahokas, le travail de traduction et de présentation dans les essais est fait par de jeunes écrivains qui ont entamé leur carrière à la fin des années cinquante ou dans les années soixante. Vu sous cet angle, l'élargissement de l'horizon est le projet d'une génération spécifique, et l'on peut présupposer qu'il sert ses intentions (je reviendrai sur cette question plus tard). 
11 On peut encore constater qu'une grande partie des essais consacrés aux précurseurs du début du siècle proposent une approche historique et même érudite du sujet. Cela est naturellement très visible dans les textes provoqués par la disparition de grands noms comme Tristan Tzara et André Breton, mais également dans les essais de Väinö Kirstinä sur les mouvements contemporains et dans les traductions de Pentti Saarikoski. L'expertise de l'histoire littéraire et des langues s'alliait au travail créatif, et ce malgré le mode de vie bohême qui était caractéristique des jeunes poètes de l'époque. D'autre part, il faut souligner que la traduction et le travail de présentation des écrivains étrangers dans des essais étaient souvent accompagnés d'une réflexion critique et créative. Dans son texte de 1965 sur le concrétisme suédois, Tom Sandell n'a pas hésité à mettre en doute les résultats des poètes qu'il présentait au public finlandais. Un exemple d'un autre type est le recueil Nollapiste d'Osmo Jokinen de 1964 qui ne consistait qu'en des pages vides où des points interrompent de temps en temps la continuité de l'espace blanc. Selon Jokinen, le recueil était une réponse et un prolongement au Degré zéro de l'écriture de Roland Barthes, dont il avait entendu parler dans les colonnes du journal Helsingin Sanomat (Kantola, 2004).

\section{Kirstinä le passeur}

12 Pour mieux cerner les enjeux de cette internationalisation du champ littéraire, on peut analyser plus en détail le travail de Väinö Kirstinä. Avec Pentti Saarikoski, il était, aux yeux du public de l'époque, le jeune écrivain qui montrait à la poésie la voie à suivre, mais aussi un intellectuel actif par exemple dans l'important groupe pacifiste appelé le « comité des $100 »$ (Sadankomitea). Le jeune essayiste Pekka Tarkka, qui deviendra plus tard le grand critique de sa génération dans le journal Helsingin Sanomat, considérait Kirstinä comme le primus motor « d'une ligne qui cherchait à défaire la langue et la réalité » et dont « le point de départ était le surréalisme et Apollinaire » (Tarkka, 1984 [1971], p. 149). Pour Tarkka, le fait que Kristinä jouait avec les mots et la typographie et ouvrait son discours poétique à tous les registres de la langue et à tous les systèmes symboliques de la société suffisait pour le qualifier de «dadaïste » (voir Tarkka, 1984 [1965], p. 99-101).

13 Comme on le voit, cette interprétation du dadaïsme et aussi, dans une certaine mesure, du surréalisme est loin de la théorie influente (bien qu'aujourd'hui fort critiquée) de Peter Bürger, qui souligne l'aspect critique de Dada, ses efforts pour saborder l'institution et les conventions de l'art (Bürger, 1984). La littérature et les arts finlandais ont connu des scandales et des provocations au cours des années soixante, mais, dans ces événements, les avant-gardes historiques n'ont pas joué le rôle d'inspirateur ou de point de référence. Les œuvres et les performances qui ont choqué le public et déclenché des procès s'ancraient plutôt dans le discours de l'art et de la littérature contemporains, comme le Pop Art, le happening et la poésie beat de Ginsberg, ou bien utilisaient des moyens de représentation du modernisme établi pour traiter des sujets considérés comme blasphématoires ${ }^{6}$. Dans l'interprétation de Kirstinä (voir surtout 1963a), qui a fortement influencé l'époque, le dadaïsme était vu comme un élément dans le continuum historique de la poésie ludique et absurde (dans la signification de «nonsense »). Ce continuum remontait aux comptines et incantations, incluait des écrivains aussi dissemblables que les prédadaïstes finnois J. L. Runeberg et Jaakko Juteini, tous deux des poètes du début du XIX ${ }^{e}$ siècle, le « véritable dadaïste » de 
Finlande Gunnar Björling et l'allemand Christian Morgenstern et finissait par les concrétistes suédois et le lettrisme. Le facteur commun de ces écrivains était la volonté de rompre les codes esthétiques, de déformer la langue pour créer du nouveau.

Kirstinä considérait donc Dada comme un mouvement esthétique et situait le travail d'expérimentation des dadaïstes sur le plan de l'innovation et de la déconstruction des conventions du discours poétique. Cette approche est évidente aussi dans son intérêt pour Charles Baudelaire, qu'il considérait comme l'auteur-clé du modernisme, et pour le surréalisme, qui était pour lui, dans sa volonté de changer le monde par la poésie, le dernier avatar ou "spasme " du romantisme. Selon Kirstinä, Baudelaire a cherché à percevoir et à exprimer la beauté et les aspects poétiques de la vie moderne contemporaine. Cette recherche a conduit la poésie à une expansion du vocabulaire et du répertoire des moyens d'expression. Elle a été essentielle aussi au surréalisme qui partage avec Baudelaire le même intérêt pour le spirituel, le nouveau, l'irrationnel et le surnaturel ; les autres «ismes »-comme le vorticisme, le futurisme et le dadaïsme peuvent être subsumés sous cette ligne générale qui constitue, avec l'art pour l'art, les deux axes fondamentaux de la poésie moderne (Kirstinä, 1963b et 1963c)7 .

Cette volonté de tracer de grandes lignes d'évolution peut surprendre de la part d'un écrivain que ses contemporains considéraient comme un destructeur de la langue et de la réalité. On peut en effet trouver dans les textes de Kirstinä des formulations qui semblent franchement paradoxales, par exemple, la caractérisation de Jyrki Pellinen, un des jeunes poètes prometteurs des années soixante, comme « un excellent avantgardiste et un bon traditionaliste " (Kirstinä, 1963d, p. 288). La clé du paradoxe réside en grande partie dans la vision que Kirstinä avait de la fonction ou de la raison d'être de la poésie moderne, d'une part, et de la situation de la Finlande par rapport à l'évolution de la poésie européenne et mondiale d'autre part. Pour lui, la poésie moderne cherche à être une expression totale et sans résidu de l'homme contemporain. Ce but l'oblige à puiser dans tout le vocabulaire existant et dans tous les discours et systèmes de communication, mais il n'est pas nécessairement antagoniste par rapport au passé et ne conduit pas, par exemple, à une purification des archaïsmes, la bête noire des modernistes des années cinquante. Expérimentation et tradition ne s'excluent pas. L'impression contraire était due, selon Kirstinä, au fait que la Finlande n'avait pas suivi l'évolution de la poésie moderne européenne et mondiale. À cause de ce retard, le renouvellement $\mathrm{du}$ discours poétique finlandais après les années quarante - par exemple, l'abandon des rimes et des systèmes métriques - a été brusque et a ressemblé à une révolution, ce que Kirstinä regrettait vivement (Kirstinä, 1962b).

Il faut souligner que, dans les essais de Kirstinä, la Finlande n'est pas située dans une périphérie réceptrice des influences, en tout cas pas d'une manière univoque. L'intérêt que Kirstinä porte à la tradition de la poésie moderne et d'avant-garde européenne et mondiale est d'abord motivé par l'évolution de la société et des rôles que la poésie peut y jouer. C'est la question « Est-ce que la poésie est toujours possible? » qui l'oriente vers Baudelaire, le surréalisme et les autres "ismes", mais cette question est en premier lieu posée par et dans la société finlandaise. C'est aussi à travers ce prisme que Kirstinä approche les poètes et les mouvements étrangers. On peut l'accuser d'avoir négligé les aspects critiques et même nihilistes de Dada, par exemple, mais cette accusation me semble manquer d'intérêt dans la mesure où il voyait dans Dada un moyen d'élargir le champ du discours poétique, y compris dans sa fonction critique par rapport à la société finlandaise des années soixante, cette fonction ayant été rendue 
possible par l'existence même de l'institution littéraire, dont il est devenu un membre incontesté8. Au lieu d'influence du centre sur la périphérie ou de mauvaise compréhension du centre par la périphérie, il vaut mieux donc caractériser le mécanisme comme un processus de transfert et d'adaptation ou de refonctionnalisation des formes d'écriture où la périphérie est un acteur créatif, agissant selon ses propres intentions et motivations.

\section{La professionnalisation du métier et la construction de l'avant-garde en Finlande}

17 Kirstinä a dû apprendre de nouvelles langues pour comprendre l'évolution de la poésie moderne depuis Baudelaire. Il a bien rentabilisé ses efforts en se profilant au cours des années soixante comme un spécialiste des avant-gardes et de la théorie poétique. Son introduction au Premier manifeste du surréalisme est éloquente dans ce sens : c'est un texte écrit par un érudit qui assume pleinement son rôle de créateur. Plus tard, Kirstinä, qui venait de la campagne profonde, était le seul parmi ses frères et sœurs à étudier à l'université et voulait devenir instituteur, a fini par enseigner la littérature à l'université aux futurs écrivains et érudits.

Cette trajectoire est un bel exemple de la professionnalisation du métier d'écrivain. Les années soixante voient une évolution des systèmes de soutien financier aux écrivains. En même temps, les écrivains ont accès à plusieurs modes de communication et à plusieurs métiers dans la production culturelle : la radio, le cinéma et la télévision emploient des écrivains, qui changent de métier d'une manière flexible, passent de la peau du critique dans celle du traducteur, du reporter ou du scénariste. Les compétences et expériences professionnelles, comme le fait d'avoir étudié à l'étranger et la connaissance des langues, sont un atout majeur dans ce monde en mutation. En témoignent les quatrièmes de couverture et autres paratextes qui offrent d'amples informations sur les parcours des écrivains. La connaissance des littératures d'avantgarde et une place visible dans les rangs des poètes novateurs constituent également un capital symbolique sur ce marché du travail. De son côté, l'institution littéraire est prête à offrir des postes d'importance aux jeunes écrivains contestataires. Par exemple, Jarkko Laine, un des membres les plus importants de l'underground de la fin des années soixante, fut recruté comme secrétaire de rédaction à Parnasso en 1969, à l'âge de 22 ans, deux ans après ses débuts et quatre ans après avoir mené une vie de beatnik à Stockholm (Haavikko, 1991, p. 141-144).

19 Le dialogue entre la poésie finlandaise et la littérature étrangère dans les années soixante était un jeu complexe d'échanges motivé tout aussi bien par l'attrait de la production expérimentale dans les autres pays que par la situation des écrivains en Finlande. Le transfert et l'adaptation de nouvelles formes d'écriture et de nouveaux intertextes étaient inséparables des mutations économiques et sociales qui conditionnaient la possibilité pour les écrivains d'exercer leur métier, mais, d'autre part, ce sont précisément les textes étrangers appartenant à des courants et mouvements contestataires qui ont permis aux écrivains finlandais de porter un nouveau regard critique sur la littérature et la société. Le dadaïsme, le surréalisme et les autres "ismes" ont été, en Finlande, la création des écrivains finlandais et de la société finlandaise, bien qu'ils s'enracinent, aussi bien sur le plan de l'écriture que sur 
les plans politique et éthique, dans la tradition internationale d'avant-garde, dont ils poursuivent le programme critique dans un nouveau contexte social et littéraire.

\section{BIBLIOGRAPHIE}

ANHAVA Tuomas, 1967, “Lyriikkakilpailumme”, Parnasso, 4, pp. 145-146.

BÜRGER Peter, 1984, Theory of the Avant-Garde, traduit de l'allemand par Michael Shaw, Minneapolis: University of Minnesota Press.

CALINESCU Matei, 1987, Five Faces of Modernity: Modernism, Avant-Garde, Decadence, Kitsch, Postmodernism, Durham: Duke University Press.

FOSTER Hal, 1994, “What's Neo about the Neo-Avant-Garde?”, October, 70, pp. 5-32.

HAAPALA Vesa, 2007, « Kokeellinen kirjallisuus ja kirjallinen vastarinta Suomessa - kiintopisteenä 1960-luku », in KATAJAMÄKI Sakari et VEIVo Harri, Kirjallisuuden avantgarde ja kokeellisuus, Helsinki : Gaudeamus, 2007, pp. 277-304.

HAAVIKKO Ritva (dir.), 1991, Miten kirjani ovat syntyneet 3, Helsinki : WSOY.

HORMia Osmo, 1962, « 60-luvun runous - mitä se on oleva ? », Parnasso, 7, pp. 293-298.

HÖKКÄ Tuula, 1999, Modernismi : uusi alku - vanhan valtaus », in LASSILA Pertti, Rintamakirjeistä tietoverkkoihin. Suomen kirjallisuushistoria 3, Helsinki : SKS, pp. 68-89.

KANTOLA Janna, 2004, « Jälkisanat naword », in JOKINEN Osmo, Nollapiste nulpunt, Nijmegen : Martin Frijns \& Vantilt, pas de pagination.

KANTOLA Janna et RIIKONEN H.K., 2007, « Käännösten merkitys suomalaiselle modernismille », in RiıKonen H.K., Kovala Urpo, KuJAMÄKi Pekka et PALOPoski Outi, Suomennoskirjallisuuden historia I, Helsinki : SKS, pp. 446-460.

KIRSTINÄ Väinö, 1962a, « Lähiperinne », Suomalainen Suomi, 7, pp. 448-449.

KIRSTINÄ Väinö, 1962b, « Kirjoittamisen ongelmat », Nuori voima, 9, p. 4.

KIRSTINÄ Väinö, 1963a, « Loruista lettrismiin », Parnasso, 8, pp. 357-365.

KIRSTINÄ Väinö, 1963b, « Mikä modernissa runoudessa on modernia ? », Nuori voima, 6, p. 4.

KIRSTINÄ Väinö, 1963c, « Surrealistit ja realistit », Suomalainen Suomi, 6, pp. 388-391.

KIRSTINÄ Väinö, 1963d, « Kielen tragedia », Suomalainen Suomi, 4, pp. 286-288.

KoSKELA Lasse, 1999, « Täyttä nykyaikaa », in RoJolA, Lea, Järkiuskosta vaistojen kapinaan, Suomen kirjallisuushistoria 2, Helsinki : SKS, pp. 259-280.

LOUNELA Pekka et MäNTYlä Jyrki, 1970, Huuto ja meteli, Hämeenlinna : Karisto.

PAAVOLAINEN Olavi, 2002 [1929], Nykyaikaa etsimässä, Helsinki : Otava.

PeltomÄKi Tauno, 1963, « Avartuva modernismi », Nuori Voima, 2-3, p. 3. 
PolAMERI Veikko, 1969, « 70-luvulle », Parnasso, 5, pp. 311-314.

ROBERTS John, 2010, "Revolutionary Pathos, Negation, and the Suspensive Avant-Garde", New Literary History, 41, pp. 717-730.

Runoseminaari, Turku : Kustannusliike Tajo, 1963.

SAARIKOSKI Pentti, 1960, « 50-luvun kasvot », Parnasso, 1, pp. 3-10.

SADIK-OGLI Nikolai, 2000, "Bloody Might: Dada in Finland in the 1920's", Journal of Finnish Studies, vol. 4, no 1, pp. 37-51.

Salo Arvo, 1960, « 50-luvun kasvot », Parnasso, 1, pp. 3-10.

SALo Arvo, 1962, « 60-luvun runous - mitä sen tulisi olla », Parnasso, 7, pp. 299-303.

SUURPÄÄ Matti, 2008, Kuljin missä kuljin, Helsinki : Otava.

TARKKA Pekka, 1984, Sanat sanoista: arvosteluja ja kirjoituksia 1957-1984, Helsinki : Otava.

VeIVo Harri et KANTOLA Janna, 2005, « Une avant-garde à deux vitesses : Dada et les littératures de la Finlande ", in BÉHAR Henri et Dufour Catherine, Dada circuit total, Lausanne : L'Âge d'Homme, p. 349-359.

VeIVo Harri, 2011, “'Broken clouds - also by instalments' - Mediating art and the everyday, the high and the low in the Finnish literary avant-garde of the 1960s", in BRU Sascha, VAN NIJS Laurence, HJARTARSON Benedikt, Nicholls Peter, ØRUM Tania et VAN DEN BERG Hubert, Regarding the Popular: High and Low Culture in the Avant-Garde and Modernism, Berlin: Walter de Gruyter, pp. 246-258.

VIIKARI Auli, 1992, « Ei kenenkään maa: 1950-luvun tropologiaa », in MAKKONEN Anna, Avoin ja suljettu: Kirjoituksia 1950-luvusta suomalaisessa kulttuurissa, Helsinki : SKS, pp. 30-77.

\section{NOTES}

1. Björling a été redécouvert et reconnu en tant que précurseur important après la guerre en Suède et, par un jeu de ricochet, dans les années soixante en Finlande. Sur le dadaïsme finlandais, voir Veivo et Kantola, 2005 et Sadik-Ogli, 2000 ; sur le vitalisme et l'expressionnisme, voir Koskela, 1999, p. 267-272.

2. Sur la continuité de l'avant-garde et la négociation entre les tendances critiques et révolutionnaires et les demandes pragmatiques de la société, voir Roberts, 2010.

3. Le séminaire de poésie de Turku fut le théâtre d'un des actes de provocation les plus célèbres de l'époque lorsque le poète Pentti Saarikoski arriva dans la salle, ivre, accompagné de sa maîtresse, ivre également, et porteur d'un revolver. Voir Runoseminaari, Hormia, 1962 et Salo, 1962.

4. Une autre solution majeure était l'engagement politique, qui deviendra de plus en plus dominant au cours de la décennie.

5. Dans la première traduction, parue dans Parnasso, les expressions obscènes étaient remplacées par un X. Cette publication fut suivie en 1963 d'un tirage limité destiné aux spécialistes. Le véritable scandale éclata en 1969 lorsque Yle (la radio-télévision finlandaise), suite à une erreur de programmation, diffusa le poème sur les deux chaînes nationales en même temps. Voir Lounela et Mäntylä, 1970. 
6. Dans la première catégorie figurent les procès contre le peintre Harro Koskinen (1969), l'écrivain-artiste Mattijuhani Koponen (1968) et l'affaire de l'émission radiophonique de « Howl » de Ginsberg, dans la deuxième le procès contre l'écrivain Hannu Salama (1964-1967).

7. L'esquisse est en fait plus complexe dans le sens où Kirstinä finit son texte (1963b) par une distinction entre le surréalisme, la poésie « qui s'intéresse à la modernité » et " l'art pour l'art ». Il me semble cependant que, dans l'ensemble du texte, la parenté du surréalisme et de la poésie moderne d'origine baudelairienne est prépondérante.

8. Voir l'analyse des poèmes du recueil Luonnollinen tanssi dans Veivo 2011.

\section{RÉSUMÉS}

L'article analyse le dialogue entre la poésie finlandaise (de langue finnoise), l'avant-garde historique et la littérature expérimentale contemporaine européenne et américaine dans les années soixante. Il s'agit d'un phénomène à facettes multiples, dont la complexité va bien au-delà de la simple arrivée tardive de nouvelles idées dans un pays lointain qui souffre du décalage de la périphérie par rapport au centre. Dans les années soixante, la poésie finlandaise cherche à sauvegarder une partie de la force critique de l'avant-garde historique et contemporaine, tout en l'adaptant au contexte local et en négociant sa position au sein d'une société de consommation et du spectacle émergente. L'intérêt que les poètes de l'époque ont porté à la littérature étrangère témoigne de l'internationalisation de la culture et de la société finlandaises, mais il est également motivé par un jeu de positionnement à l'intérieur du champ littéraire national, qui connaît au cours de la décennie une série de mutations fondamentales. La carrière du poète Väinö Kirstinä servira d'étude de cas dans l'analyse de ces relations.

The article analyses the dialogue between Finnish poetry, the historical avant-garde and contemporary experimental literature in Europe and the U.S. in the 1960s. This complex dialogue cannot be reduced to explanatory models based on the assumption of a developmental lag between the center and the periphery. In the 1960s, Finnish poetry seeks to maintain the critical force of the historical and contemporary avant-garde, adapting it into the local context and negotiating at the same time its own place in a rapidly developing society of consumption and spectacle. The Finnish writers' interest towards foreign literature testifies of the internationalization of Finnish culture and society, but it is also motivated by mutations of the literary field in Finland. The poet Väinö Kirstinä's career serves as a case study that permits to analyse global tendencies at the level of the individual.

Artikkeli tarkastelee suomalaisen runouden, historiallisen avant-garden ja kokeellisen eurooppalaisen ja amerikkalaisen runouden dialogia 1960-luvulla. Kyseessä on monitahoinen ilmiö, joka ei ole palautettavissa periferian jälkijättöisyyttä korostaviin selitysmalleihin. 60-luvun suomalainen runous pyrkii säilyttämään avantgarden kriittisen ulottuvuuden ja soveltamaan sen paikalliseen kontekstiin; samalla se neuvottelee omasta asemastaan nopeasti kehittyvässä kulutus- ja spektaakkeliyhteiskunnassa. Aikakauden runoilijoiden kiinnostus ulkomaista kirjallisuutta kohtaan on osoitus suomalaisen yhteiskunnan kansainvälistymisestä, mutta myös strategisista valinnoista muuttuvan kansallisen kirjallisen kentän sisällä. Väinö Kirstinän uraa tarkastellaan tapaustutkimuksena, joka auttaa hahmottamaan yleisiä muutoksia yksilön tasolla. 
INDEX

Keywords : Avant-garde, Comparative Literature, Finland, Poetry, Twentieth century, The 1960s, Finnish literature, Translation

Thèmes : littérature finnoise, littérature comparée

Index géographique : Finlande, Japon, Lahti, Stockholm, Turku

motsclesfi runous, avant-garde, kääntäminen, 1900-luku, 1960-luvut, Suomen kirjallisuus,

kirjallisuustiede

Mots-clés : poésie, avant-garde, traduction

Index chronologique : XXe siècle (milieu), XXe siècle 